\begin{tabular}{|c|c|}
\hline Title & Time O perators of a Hamiltonian with Purely Discrete Spectrum \\
\hline Author(s) & A rai, A sao; Matsuzawa, Y asumichi \\
\hline Citation & $\begin{array}{l}\text { Reviews in Mathematical Physics, 20(8), 951-978 } \\
\text { https://doi.org/10.1142/50129055X 08003481 }\end{array}$ \\
\hline Issue Date & $2008-09$ \\
\hline Doc URL & http:/hdl.handle.net $/ 2115 / 38104$ \\
\hline Rights & $\begin{array}{l}\text { Electronic version of an article published as Reviews in Mathematical Physics, V ol. 20, Issue. 8, 2008, pp.951-978, } \\
\text { DOI:10.1142/f012955X 08003481 @ W orld Scientific Publishing Company, } \\
\text { http://www.worldscinet.com/rmp/mp.shtml }\end{array}$ \\
\hline Type & article (author version) \\
\hline File Information & ArMa rev3.pdf \\
\hline
\end{tabular}

Instructions for use 


\title{
Time Operators of a Hamiltonian with Purely Discrete Spectrum
}

\author{
Asao Arai ${ }^{*}, 1$ and Yasumichi Matsuzawa ${ }^{2}$ \\ Department of Mathematics, Hokkaido University \\ Sapporo 060-0810 \\ Japan \\ ${ }^{1}$ E-mail: arai@math.sci.hokudai.ac.jp \\ 2 E-mail: s073035@math.sci.hokudai.ac.jp
}

\begin{abstract}
We develop a mathematical theory of time operators of a Hamiltonian with purely discrete spectrum. The main results include boundedness, unboundedness and spectral properties of them. In addition, possible connections of a time operator of $H$ with regular perturbation theory are discussed.
\end{abstract}

Keywords. canonical commutation relation, Hamiltonian, time operator, time-energy uncertainty relation, phase operator, spectrum, regular perturbation theory.

Mathematics Subject Classification (2000). 81Q10, 47N50.

\section{Introduction}

This paper is concerned with mathematical theory of time operators in quantum mechanics $[2,3,4,6,12]$. There are some types of time operators which are not necessarily equivalent each other. For the reader's convenience, we first recall the definitions of them with comments.

Let $\mathcal{H}$ be a complex Hilbert space. We denote the inner product and the norm of $\mathcal{H}$ by $\langle\cdot, \cdot\rangle$ (antilinear in the first variable) and $\|\cdot\|$ respectively. For a linear operator $A$ on a Hilbert space, $D(A)$ denotes the domain of $A$.

Let $H$ be a self-adjoint operator on $\mathcal{H}$ and $T$ be a symmetric operator on $\mathcal{H}$.

The operator $T$ is called a time operator of $H$ if there is a (not necessarily dense) subspace $\mathcal{D} \neq\{0\}$ of $\mathcal{H}$ such that $\mathcal{D} \subset D(T H) \cap D(H T)$ and the canonical commutation relation $(\mathrm{CCR})$

$$
[T, H]:=(T H-H T)=i
$$

${ }^{*}$ Corresponding author. The work is supported by the Grant-in-Aid No.17340032 for Scientific Research from Japan Society for the Promotion of Science (JSPS). 
holds on $\mathcal{D}$ (i.e., $[T, H] \psi=i \psi, \forall \psi \in \mathcal{D}$ ), where $i$ is the imaginary unit. In this case, $T$ is called a canonical conjugate to $H$ too.

The name "time operator" for the operator $T$ comes from the quantum mechanical context where $H$ is taken to be the Hamiltonian of a quantum system and the heuristic classical-quantum correspondence based on the structure that, in the classical relativistic mechanics, time is a canonical conjugate variable to energy in each Lorentz frame of coordinates. Note also that the dimension of $T$ is that of time if the dimension of $H$ is that of energy in the original unit system where the right hand side of (1.1) takes the form $i \hbar$ with $\hbar$ being the Planck constant $h$ divided by $2 \pi$. We remark, however, that this name is somewhat misleading, because, in the framework of the standard quantum mechanics, time is not an observable, but just a parameter assigning the time when a quantum event is observed. But we follow the convention in this respect. By the same reason as just remarked, $T$ is not necessarily (essentially) self-adjoint. But this does not mean that it is "unphysical" $[2,12]$. Note also that we do not require the denseness of the subspace $\mathcal{D}$ in the definition stated above. This is more general. In fact, there is an example of the pair $(T, H)$ satisfying $(1.1)$ on a non-dense subsapce $\mathcal{D}[8,10]$.

From a representation theoretic point of view, the pair $(T, H)$ is a symmetric representation of the CCR with one degree of freedom. But one should remember that, as for this original form of representation of the CCR, the von Neumann uniqueness theorem ([13], [14, Theorem VIII.14]) does not necessarily hold. In other words, $(T, H)$ is not necessarily unitarily equivalent to a direct sum of the Schrödinger representation of the CCR with one degree of freedom. Indeed, for example, it is obvious that, if $T$ or $H$ is bounded below or bounded above, then $(T, H)$ cannot be unitarily equivalent to a direct sum of the Schrödinger representation of the CCR with one degree of freedom.

A classification of pairs $(T, H)$ with $T$ being a bounded self-adjoint operator has been done by G. Dorfmeister and J. Dorfmeister [7]. We remark, however, that the class discussed in [7] does not cover the pairs $(T, H)$ considered in this paper, because the paper [7] treats only the case where $T$ is bounded and absolutely continuous.

A weak form of time operator is defined as follows. We say that a symmetric operator $T$ is a weak time operator of $H$ if there is a subspace $\mathcal{D}_{\mathrm{w}} \neq\{0\}$ of $\mathcal{H}$ such that $\mathcal{D}_{\mathrm{w}} \subset$ $D(T) \cap D(H)$ and

$$
\langle T \psi, H \phi\rangle-\langle H \psi, T \phi\rangle=\langle\psi, i \phi\rangle, \quad \psi, \phi \in \mathcal{D}_{\mathrm{w}},
$$

i.e., $(T, H)$ satisfies the CCR in the sense of sesquilinear form on $\mathcal{D}_{\mathrm{w}}$. Obviously a time operator $T$ of $H$ is a weak time operator of $H$. But the converse is not true (it is easy to see, however, that, if $T$ is a weak time operator of $H$ and $\mathcal{D}_{\mathrm{w}} \subset D(T H) \cap D(H T)$, then $T$ is a time operator). An important aspect of a weak time operator $T$ of $H$ is that a time-energy uncertainty relation is naturally derived [2, Proposition 4.1]: for all unit vectors $\psi$ in $\mathcal{D}_{\mathrm{w}} \subset D(T) \cap D(H)$,

$$
(\Delta T)_{\psi}(\Delta H)_{\psi} \geq \frac{1}{2}
$$

where, for a linear operator $A$ on $\mathcal{H}$ and $\phi \in D(A)$ with $\|\phi\|=1$,

$$
(\Delta A)_{\phi}:=\|(A-\langle\phi, A \phi\rangle) \phi\|,
$$


called the uncertainty of $A$ in the vector $\phi$.

In contrast to the weak form of time operator, there is a strong form. We say that $T$ is a strong time operator of $H$ if, for all $t \in \mathbb{R}, e^{-i t H} D(T) \subset D(T)$ and

$$
T e^{-i t H} \psi=e^{-i t H}(T+t) \psi, \quad \psi \in D(T) .
$$

We call (1.2) the weak Weyl relation [2]. From a representation theoretic point of view, we call a pair $(T, H)$ obeying the weak Weyl relation a weak Weyl representation of the CCR. This type of representation of the CCR was extensively studied by Schmüdgen $[17,18]$. It is shown that a strong time operator of $H$ is a time operator of $H$ [12]. But the converse is not true. In fact, the time operators considered in the present paper are not strong ones.

There is a generalized version of strong time operator [2]. We say that $T$ is a generalized time operator of $H$ if, for each $t \in \mathbb{R}$, there is a bounded self-adjoint operator $K(t)$ on $\mathcal{H}$ with $D(K(t))=\mathcal{H}, e^{-i t H} D(T) \subset D(T)$ and a generalized weak Weyl relation (GWWR)

$$
T e^{-i t H} \psi=e^{-i t H}(T+K(t)) \psi \quad(\forall \psi \in D(T))
$$

holds. In this case, the bounded operator-valued function $K(t)$ of $t \in \mathbb{R}$ is called the commutation factor of the GWWR under consideration.

We now come to the subject of the present paper. In his interesting paper [9], Galapon showed by an explicit construction that, for every self-adjoint operator $H$ (a Hamiltonian) on an abstract Hilbert space $\mathcal{H}$ which is bounded below and has purely discrete spectrum with some growth condition, there is a time operator $T_{1}$ on $\mathcal{H}$, which is a bounded selfadjoint operator under an additional condition (for the definition of $T_{1}$, see (2.12) below). To be definite, we call the operator $T_{1}$ introduced in [9] the Galapon time operator.

An important point of Galapon's work [9] is in that it disproved the long-standing belief or folklore among physicists that there is no self-adjoint operator canonically conjugate to a Hamiltonian which is bounded below (for a historical survey, see Introduction of [9]).

Motivated by work of Galapon [9], we investigate, in this paper, properties of time operators of a self-adjoint operator $H$ with purely discrete spectrum. In Section 2, we introduce a densely defined linear operator $T$ whose restriction to a subspace yields the Galapon time operator $T_{1}$ and prove basic properties of $T$ and $T_{1}$, in particular the closedness of $T$. It follows that, if $T$ is bounded, then $T$ is self-adjoint with $D(T)=\mathcal{H}$ and a time operator of $H$. We denote by $T^{\#}$ one of $T_{1}, T$ and $T^{*}$ (the adjoint of $T$ ). In Section 3, we discuss some general properties of $T^{\#}$. Moreover the reflection symmetry of the spectrum of $T^{\#}$ with respect to the imaginary axis is proved. Sections $4-6$ are the main parts of this paper. In Section 4, we present a general criterion for $T$ to be bounded with $D(T)=\mathcal{H}$, while, in Section 5, we give a sufficient condition for $T$ to be unbounded. In Section 6, we present a necessary and sufficient condition for $T$ to be Hilbert-Schmidt. In Section 7, we show that, under some condition, the Galapon time operator is a generalized time operator of $H$, too. We also discuss non-differentiability of the commutation factor $K$ in the GWWR for $\left(T_{1}, H\right)$. In the last section, we consider a perturbation of $H$ by a symmetric operator and try to draw out physical meanings of $T_{1}$ and $K$ in the context of regular perturbation theory. 


\section{Time Operators}

In this section, we recapitulate some basic aspects of the Galapon time operator in more apparent manner than in [9].

Let $\mathcal{H}$ be a complex Hilbert space and

$H$ be a self-adjoint operator on $\mathcal{H}$ which has the following properties (H.1) and (H.2):

(H.1) The spectrum of $H$, denoted $\sigma(H)$, is purely discrete with $\sigma(H)=\left\{E_{n}\right\}_{n=1}^{\infty}$, where each eigenvalue $E_{n}$ of $H$ is simple and

$$
0<E_{n}<E_{n+1}
$$

for all $n \in \mathbb{N}$ (the set of positive integers).

(H.2) $\sum_{n=1}^{\infty} \frac{1}{E_{n}{ }^{2}}<\infty$.

Throughout the present paper we assume (H.1) and (H.2).

Remark 2.1 The positivity condition $E_{n}>0$ for the eigenvalues of $H$ does not lose generality, because, if $H$ is bounded below, but not strictly positive, then one needs only to consider, instead of $H, \tilde{H}:=H+c$ with a constant $c>-\inf \sigma(H)$, which is a strictly positive self-adjoint operator.

Property (H.2) implies that

$$
E_{n} \rightarrow \infty \quad(n \rightarrow \infty)
$$

Let $e_{n}$ be a normalized eigenvector of $H$ belonging to eigenvalue $E_{n}$ :

$$
H e_{n}=E_{n} e_{n}, \quad n \in \mathbb{N} .
$$

Then, by property (H.1), the set $\left\{e_{n}\right\}_{n=1}^{\infty}$ is a complete orthonormal system (C.O.N.S.) of $\mathcal{H}$.

\section{Lemma 2.1}

(i) For all $m \in \mathbb{N}$,

$$
\sum_{n \neq m}^{\infty} \frac{1}{\left(E_{n}-E_{m}\right)^{2}}<\infty .
$$

In particular, for each $m \in \mathbb{N}$,

$$
\sum_{n \neq m}^{\infty} \frac{1}{E_{n}-E_{m}} e_{n}
$$

converges in $\mathcal{H}$. 
(ii) For all $n \in \mathbb{N}$ and vectors $\psi$ in $\mathcal{H}$, the infinite series

$$
\sum_{m \neq n}^{\infty} \frac{\left\langle e_{m}, \psi\right\rangle}{E_{n}-E_{m}}
$$

absolutely converges.

Proof. (i) By (2.1), we have

$$
C_{m}:=\sup _{n \neq m} \frac{1}{\left(1-\frac{E_{m}}{E_{n}}\right)^{2}}<\infty .
$$

Hence we have

$$
\sum_{n \neq m}^{\infty} \frac{1}{\left|E_{n}-E_{m}\right|^{2}} \leq C_{m} \sum_{n \neq m}^{\infty} \frac{1}{E_{n}^{2}}<\infty .
$$

(ii) By the Cauchy-Schwarz inequality, the Parseval equality and part (i), we have

$$
\begin{aligned}
\sum_{m \neq n}^{\infty}\left|\frac{\left\langle e_{m}, \psi\right\rangle}{E_{n}-E_{m}}\right| & \leq\left(\sum_{m \neq n}^{\infty}\left|\left\langle e_{m}, \psi\right\rangle\right|^{2}\right)^{\frac{1}{2}}\left(\sum_{m \neq n}^{\infty}\left|\frac{1}{E_{n}-E_{m}}\right|^{2}\right)^{\frac{1}{2}} \\
& \leq\|\psi\|\left(\sum_{m \neq n}^{\infty}\left|\frac{1}{E_{n}-E_{m}}\right|^{2}\right)^{\frac{1}{2}}<\infty
\end{aligned}
$$

By Lemma 2.1-(ii), one can define a linear operator $T$ on $\mathcal{H}$ as follows:

$$
\begin{aligned}
D(T) & :=\left\{\left.\psi \in \mathcal{H}\left|\sum_{n=1}^{\infty}\right| \sum_{m \neq n} \frac{\left\langle e_{m}, \psi\right\rangle}{E_{n}-E_{m}}\right|^{2}<\infty\right\}, \\
T \psi & :=i \sum_{n=1}^{\infty}\left(\sum_{m \neq n}^{\infty} \frac{\left\langle e_{m}, \psi\right\rangle}{E_{n}-E_{m}}\right) e_{n}, \quad \psi \in D(T) .
\end{aligned}
$$

Note that

$$
\|T \psi\|^{2}=\sum_{n=1}^{\infty}\left|\sum_{m \neq n}^{\infty} \frac{\left\langle e_{m}, \psi\right\rangle}{E_{n}-E_{m}}\right|^{2} .
$$

For a subset $\mathcal{D} \subset \mathcal{H}$, we denote by l.i.h.( $\mathcal{D})$ the subspace algebraically spanned by the vectors of $\mathcal{D}$.

The subspace

$$
\mathcal{D}_{0}:=\text { l.i.h. }\left(\left\{e_{n}\right\}_{n=1}^{\infty}\right)
$$

is dense in $\mathcal{H}$. 
Lemma 2.2 The operator $T$ is densely defined with $\mathcal{D}_{0} \subset D(T)$ and

$$
T e_{k}=i \sum_{n \neq k}^{\infty} \frac{1}{E_{n}-E_{k}} e_{n}, \quad k \in \mathbb{N} .
$$

Proof. To prove $\mathcal{D}_{0} \subset D(T)$, it is sufficient to show that $e_{k} \in D(T), k \in \mathbb{N}$. Putting

$$
c_{n}(k):=\sum_{m \neq n}^{\infty} \frac{\left\langle e_{m}, e_{k}\right\rangle}{E_{n}-E_{m}}
$$

we have $c_{k}(k)=0$ and $c_{n}(k)=1 /\left(E_{n}-E_{k}\right)$ for $n \neq k$. Hence, by Lemma 2.1-(i), we have

$$
\sum_{n=1}^{\infty}\left|c_{n}(k)\right|^{2}=\sum_{n \neq k}^{\infty} \frac{1}{\left(E_{n}-E_{k}\right)^{2}}<\infty .
$$

Hence $e_{k} \in D(T)$ and (2.11) holds.

In general, it is not clear whether or not $T$ is a symmetric operator. But a restriction of $T$ to a smaller subspace gives a symmetric operator. Indeed, we have the following fact:

Lemma 2.3 ([9]) The operator

$$
T_{1}:=T \mid \mathcal{D}_{0}
$$

(the restriction of $T$ to $\mathcal{D}_{0}$ ) is symmetric.

Proof. It is enough to show that, for all $\psi \in \mathcal{D}_{0},\langle\psi, T \psi\rangle$ is real. For a complex number $z \in \mathbb{C}$ (the set of complex numbers), we denote its complex conjugate by $z^{*}$. We have

$$
\langle\psi, T \psi\rangle=i \sum_{n=1}^{\infty}\left\langle\psi, e_{n}\right\rangle \sum_{m \neq n}^{\infty} \frac{\left\langle e_{m}, \psi\right\rangle}{E_{n}-E_{m}} .
$$

Hence

$$
\langle\psi, T \psi\rangle^{*}=i \sum_{n=1}^{\infty}\left\langle e_{n}, \psi\right\rangle \sum_{m \neq n}^{\infty} \frac{\left\langle\psi, e_{m}\right\rangle}{E_{m}-E_{n}} .
$$

Since $\psi$ is in $\mathcal{D}_{0}$, the double sum on $m, n$ with $m \neq n$ is a sum consisting of a finite term. Hence we can exchange the sum on $n$ and that on $m$ to obtain

$$
\langle\psi, T \psi\rangle^{*}=i \sum_{m=1}^{\infty}\left\langle\psi, e_{m}\right\rangle \sum_{n \neq m}^{\infty} \frac{\left\langle e_{n}, \psi\right\rangle}{E_{m}-E_{n}}=\langle\psi, T \psi\rangle .
$$

Hence $\langle\psi, T \psi\rangle$ is real.

The operator $T_{1}$ defined by (2.12) is the time operator introduced by Galapon in [9]. Obviously we have

$$
T_{1} \subset T
$$

Hence

$$
T^{*} \subset T_{1}^{*} \text {. }
$$


Remark 2.2 It is asserted in [9] that $T_{1}$ is essentially self-adjoint without additional conditions. But, unfortunately, we find that this is not conclusive, because the proof of it given in [9] (pp.2678-2679) has some gap: the interchange of the double sum in Equation (2.30) on p.2678 in [9] may not be justified, at least, by the reasoning given there. The assertion is true in the case where $T_{1}$ becomes a bounded operator under an additional condition for $\left\{E_{n}\right\}_{n=1}^{\infty}$, as we show below in the present paper. But, in the case where $T_{1}$ is unbounded, it seems to be very difficult to prove or disprove the essential self-adjointness of $T_{1}$. We leave this problem for future study.

Lemma 2.4 The subspace

$$
\mathcal{D}_{\mathrm{c}}:=\text { l.i.h. }\left(\left\{e_{n}-e_{m} \in \mathcal{H} \mid n, m \geq 1\right\}\right) .
$$

is dense in the Hilbert space $\mathcal{H}$. Moreover

$$
\mathcal{D}_{\mathrm{c}} \subset \mathcal{D}_{0} \subset D(T) .
$$

Proof. Let $\psi \in \mathcal{D}_{\mathrm{c}}^{\perp}$ (the orthogonal complement of $\mathcal{D}_{\mathrm{c}}$ ). Then, for all $m, n \geq 1$, $\left|\left\langle e_{n}, \psi\right\rangle\right|^{2}=\left|\left\langle e_{m}, \psi\right\rangle\right|^{2}$. By the Parseval equality, $\|\psi\|^{2}=\lim _{N \rightarrow \infty} N\left|\left\langle e_{m}, \psi\right\rangle\right|^{2}$. This implies that $\left|\left\langle e_{m}, \psi\right\rangle\right|^{2}=0$ for all $m \geq 1$ and $\|\psi\|=0$. Hence $\psi=0$. Thus $\mathcal{D}_{\text {c }}$ is dense in $\mathcal{H}$. Inclusion relation $(2.16)$ is obvious.

Theorem 2.5 ([9]) It holds that

$$
\mathcal{D}_{\mathrm{c}} \subset D\left(T_{1} H\right) \cap D\left(H T_{1}\right)
$$

and

$$
\left[T_{1}, H\right] \psi=i \psi, \quad \psi \in \mathcal{D}_{\mathrm{c}} .
$$

Theorem 2.5 shows that $T_{1}$ is a time operator of $H$.

Remark 2.3 It is easy to see that, for all $k \in \mathbb{N}, T_{1} e_{k} \notin D(H)$. Hence $\mathcal{D}_{0} \not \subset D\left(H T_{1}\right)$. Therefore one can not consider the commutation relation $\left[T_{1}, H\right]$ on $\mathcal{D}_{0}$. Moreover, by direct computation, we have

$$
\left\langle T_{1} e_{k}, H e_{\ell}\right\rangle-\left\langle H e_{k}, T_{1} e_{\ell}\right\rangle=-i\left(1-\delta_{k \ell}\right), \quad k, \ell \in \mathbb{N} .
$$

This means that $\left(T_{1}, H\right)$ does not satisfy the CCR in the sense of sesquilinear form on $\mathcal{D}_{0}$ (a weak form of the CCR), either. These facts suggest that the pair $\left(T_{1}, H\right)$ is very sensitive to the domain on which their commutation relation is applied.

In concluding this section we discuss shortly non-uniqueness of time operators of $H$. We introduce a set of symmetric operators associated with $H$ :

$$
\begin{aligned}
\{H\}_{\mathcal{D}_{c}}^{\prime}:=\{S \mid S \text { is a symmetric operator on } \mathcal{H} \text { such that } & \\
& \left.\mathcal{D}_{c} \subset D(S H) \cap D(H S) \text { and } S H \psi=H S \psi, \forall \psi \in \mathcal{D}_{c}\right\},
\end{aligned}
$$

which may be viewed as a commutant of $\{H\}$ in a restricted sense. It is easy to see that, for all real-valued continuous function $f$ on $\mathbb{R}$, the operator $f(H)$ defined via the functional calculus is in $\{H\}_{\mathcal{D}_{c}}^{\prime}$. 
Proposition 2.6 For all $S \in\{H\}_{\mathcal{D}_{c}}^{\prime}, \mathcal{D}_{c} \subset D\left(\left(T_{1}+S\right) H\right) \cap D\left(H\left(T_{1}+S\right)\right)$ and

$$
\left[T_{1}+S, H\right] \psi=i \psi, \quad \psi \in \mathcal{D}_{c} .
$$

Proof. A direct computation using Theorem 2.5 and (2.20).

Proposition 2.7 Let $T_{2}$ be a time operator of $H$ such that $\mathcal{D}_{\mathrm{c}} \subset D\left(T_{2} H\right) \cap D\left(H T_{2}\right)$ and

$$
\left[T_{2}, H\right] \psi=i \psi, \quad \forall \psi \in \mathcal{D}_{\mathrm{c}} .
$$

Then $T_{2}=T_{1}+S$ with some $S \in\{H\}_{\mathcal{D}_{c}}^{\prime}$.

Proof. We need only to show that $S:=T_{2}-T_{1}$ is in $\{H\}_{\mathcal{D}_{c}}^{\prime}$. But this is obvious.

\section{General Properties}

\subsection{Closedness of $T$ and symmetry of $T^{*}$}

Lemma $3.1 \mathcal{D}_{0} \subset D\left(T^{*}\right)$ and $T^{*} \mid \mathcal{D}_{0}=T_{1}$, i.e., $T_{1} \subset T^{*}$.

Proof. It is enough to show that, for all $k \in \mathbb{N}, e_{k} \in D\left(T^{*}\right)$ and $T^{*} e_{k}=T e_{k}\left(=T_{1} e_{k}\right)$. It is easy to see that, for all $\psi \in D(T)$,

$$
\left\langle e_{k}, T \psi\right\rangle=i \sum_{m \neq k}^{\infty} \frac{\left\langle e_{m}, \psi\right\rangle}{E_{k}-E_{m}} .
$$

By Lemma 2.2, the right hand side is equal to $\left\langle T e_{k}, \psi\right\rangle$. Hence $e_{k} \in D\left(T^{*}\right)$ and $T^{*} e_{k}=$ $T e_{k}$.

Proposition 3.2 The operator $T$ is closed and

$$
T^{*} \subset T
$$

In particular, if $T$ is bounded, then $T$ is self-adjoint with $D(T)=\mathcal{H}$.

Proof. Let $\psi_{k} \in D(T), k \in \mathbb{N}$ and $\psi_{k} \rightarrow \psi \in \mathcal{H}, T \psi_{k} \rightarrow \phi \in \mathcal{H}$ as $k \rightarrow \infty$. Then $\sup _{k \geq 1}\left\|T \psi_{k}\right\|<\infty$. Hence, by (2.9), there exists a constant $C>0$ independent of $k \in \mathbb{N}$ such that

$$
\sum_{n=1}^{\infty}\left|\sum_{n \neq m} \frac{\left\langle e_{m}, \psi_{k}\right\rangle}{E_{n}-E_{m}}\right|^{2} \leq C .
$$

By (2.6), we have

$$
\lim _{k \rightarrow \infty} \sum_{n \neq m}^{\infty} \frac{\left\langle e_{m}, \psi_{k}\right\rangle}{E_{n}-E_{m}}=\sum_{n \neq m}^{\infty} \frac{\left\langle e_{m}, \psi\right\rangle}{E_{n}-E_{m}}
$$


Hence it follows that

$$
\sum_{n=1}^{\infty}\left|\sum_{n \neq m}^{\infty} \frac{\left\langle e_{m}, \psi\right\rangle}{E_{n}-E_{m}}\right|^{2} \leq C .
$$

Therefore $\psi \in D(T)$. By (3.1) and (3.3), we have for all $\ell \in \mathbb{N}$

$$
\lim _{k \rightarrow \infty}\left\langle e_{\ell}, T \psi_{k}\right\rangle=\left\langle e_{\ell}, T \psi\right\rangle .
$$

Hence $\left\langle e_{\ell}, \phi\right\rangle=\left\langle e_{\ell}, T \psi\right\rangle, \ell \in \mathbb{N}$, implying $\phi=T \psi$. Thus $T$ is closed.

To prove (3.2), let $\psi \in D\left(T^{*}\right)$. Putting $\eta=T^{*} \psi$, we have $\langle\eta, \chi\rangle=\langle\psi, T \chi\rangle$ for all $\chi \in D(T)$. Taking $\chi=e_{k}(k \in \mathbb{N})$, we have

$$
\left\langle\eta, e_{k}\right\rangle=i \sum_{n \neq k}^{\infty} \frac{\left\langle\psi, e_{n}\right\rangle}{E_{n}-E_{k}}
$$

which implies that

$$
\sum_{k=1}^{\infty}\left|\sum_{n \neq k}^{\infty} \frac{\left\langle\psi, e_{n}\right\rangle}{E_{n}-E_{k}}\right|^{2}=\|\eta\|^{2}<\infty .
$$

Hence $\psi \in D(T)$. Then, by (3.1), the right hand side of (3.4) is equal to $\left\langle T \psi, e_{k}\right\rangle$. Hence $\eta=T \psi$. Thus $(3.2)$ holds.

Let $T$ be bounded. Then, by the denseness of $D(T)$ and the closedness of $T, D(T)=$ $\mathcal{H}$. Hence $D\left(T^{*}\right)=\mathcal{H}$. Thus, by $(3.2), T^{*}=T$, i.e., $T$ is self-adjoint.

Corollary 3.3 The operator $T^{*}$ is symmetric.

Proof. By Lemma 3.1, $T^{*}$ is densely defined. Hence, by Proposition 3.2, $T^{*} \subset T=$ $\left(T^{*}\right)^{*}$. Thus $T^{*}$ is symmetric.

Thus we have

$$
T_{1} \subset T^{*} \subset T \text {. }
$$

Corollary 3.3 shows that $T^{*}$ also is a time operator of $H$.

For a closable operator $A$ on a Hilbert space, we denote its closure by $\bar{A}$.

Proposition $3.4 \bar{T}_{1}=T^{*}$.

Proof. Note that $\bar{T}_{1}=T^{*}$ if and only if $T_{1}{ }^{*}=T$. By (3.5), we have $\bar{T}_{1} \subset T^{*}$. Hence $T \subset T_{1}{ }^{*}$. Thus it is enough to show that $D\left(T_{1}^{*}\right) \subset D(T)$. For all $\psi \in D\left(T_{1}^{*}\right)$, we have

$$
\left\langle T_{1}^{*} \psi, e_{l}\right\rangle=\left\langle\psi, T_{1} e_{l}\right\rangle=i \sum_{n \neq l} \frac{\left\langle\psi, e_{n}\right\rangle}{E_{n}-E_{l}} .
$$

Hence we obtain

$$
\infty>\left\|T_{1}^{*} \psi\right\|^{2}=\sum_{l=1}^{\infty}\left|\left\langle T_{1}^{*} \psi, e_{l}\right\rangle\right|^{2}=\sum_{l=1}^{\infty}\left|\sum_{n \neq l} \frac{\left\langle\psi, e_{n}\right\rangle}{E_{n}-E_{l}}\right|^{2},
$$

implying that $\psi \in D(T)$. Thus $D\left(T_{1}^{*}\right) \subset D(T)$. 


\subsection{Absence of invariant dense domains for $T$ under some con- dition}

We first note the following general fact:

Proposition 3.5 Let $Q$ be a bounded self-adjoint operator on $\mathcal{H}$ and $P$ be a self-adjoint operator on $\mathcal{H}$. Suppose that there is a dense subspace $\mathcal{D}$ in $\mathcal{H}$ such that the following (i)-(iii) hold:

(i) $Q \mathcal{D} \subset \mathcal{D} \subset D(P)$.

(ii) $\mathcal{D}$ is a core of $P$.

(iii) The pair $(Q, P)$ obeys the $C C R$ on $\mathcal{D}:[Q, P] \psi=i \psi, \forall \psi \in \mathcal{D}$.

Then $\sigma(P)=\mathbb{R}$.

Proof. Since $Q$ is a bounded self-adjoint operator, we have for all $t \in \mathbb{R}$

$$
e^{i t Q}=\sum_{k=0}^{\infty} \frac{(i t Q)^{k}}{k !}
$$

in operator norm. Conditions (i) and (iii) imply that, for all $k \in \mathbb{N}$ and $\psi \in \mathcal{D}$

$$
Q^{k} P \psi-P Q^{k} \psi=i k Q^{k-1} \psi
$$

Hence, for all $t \in \mathbb{R}$ and vectors $\psi$ in $\mathcal{D}$, we have

$$
\begin{aligned}
e^{i t Q} P \psi & =P \psi+\sum_{k=1}^{\infty} \frac{(i t)^{k}}{k !} Q^{k} P \psi \\
& =P \psi+\sum_{k=1}^{\infty} \frac{(i t)^{k}}{k !}\left(P Q^{k}+i k Q^{k-1}\right) \psi \\
& =P \psi+\sum_{k=1}^{\infty}\left\{P \frac{(i t Q)^{k}}{k !} \psi-t \frac{(i t Q)^{k-1}}{(k-1) !} \psi\right\} .
\end{aligned}
$$

It follows from the closedness of $P$ that $e^{i t Q} \psi$ is in $D(P)$ and

$$
P e^{i t Q} \psi=e^{i t Q} P \psi+t e^{i t Q} \psi \text {. }
$$

By condition (ii), this equality extends to all $\psi \in D(P)$ with $e^{i t Q} \psi \in D(P), \forall t \in \mathbb{R}, \forall \psi \in$ $D(P)$. Hence the operator equality $e^{-i t Q} P e^{i t Q}=P+t$ follows. Thus $\sigma(P)=\sigma(P+t)$ for all $t \in \mathbb{R}$. This implies that $\sigma(P)=\mathbb{R}$.

Theorem 3.6 If $T$ is bounded (hence self-adjoint by Proposition 3.2), then there is no dense subspace $\mathcal{D}$ in $\mathcal{H}$ such that the following (i)-(iii) hold:

(i) $T \mathcal{D} \subset \mathcal{D} \subset D(H)$. 
(ii) $\mathcal{D}$ is a core of $H$.

(iii) The pair $(T, H)$ obeys the $C C R$ on $\mathcal{D}$,

Proof. Suppose that there were such a dense subspace $\mathcal{D}$ as stated above. Then we can apply Proposition 3.5 with $(Q, P)=(T, H)$ to conclude that $\sigma(H)=\mathbb{R}$. But this is a contradiction.

Remark 3.1 A special case of this theorem was established in [7, Theorem 9.5].

\subsection{Reflection symmetry of the spectrum of $T_{1}, T^{*}$ and $T$}

We first recall the definition of the spectrum of a general linear operator (not necessarily closed). For a linear operator $A$ on a Hilbert space $\mathcal{K}$, the resolvent set of $A$, denoted $\rho(A)$, is defined by

$$
\begin{array}{r}
\rho(A):=\{z \in \mathbb{C} \mid \operatorname{Ran}(A-z) \text { (the range of } A-z) \text { is dense in } \mathcal{K} \\
\text { and } \left.A-z \text { is injective with }(A-z)^{-1} \text { bounded }\right\} .
\end{array}
$$

Then the set

$$
\sigma(A):=\mathbb{C} \backslash \rho(A)
$$

is called the spectrum of $A$. The set of eigenvalues of $A$, called the point spectrum of $A$, is denoted $\sigma_{\mathrm{p}}(A)$.

We denote by $T^{\#}$ any of $T_{1}, T^{*}$ and $T$.

We define a conjugation $J$ on $\mathcal{H}$ by

$$
J \psi:=\sum_{n=1}^{\infty}\left\langle\psi, e_{n}\right\rangle e_{n}, \quad \psi \in \mathcal{H} .
$$

Proposition 3.7 The spectrum $\sigma\left(T^{\#}\right)$ of $T^{\#}$ is reflection symmetric with respect to the imaginary axis, i.e., if $z \in \sigma\left(T^{\#}\right)$, then $-z^{*} \in \sigma\left(T^{\#}\right)$. In particular, if $T$ is self-adjoint, then $\sigma(T)$ is reflection symmetric with respect to the origin of the real axis. Moreover, for all $z \in \sigma_{\mathrm{p}}\left(T^{\#}\right),-z^{*}$ is in $\sigma_{\mathrm{p}}\left(T^{\#}\right)$ and

$$
J \operatorname{ker}\left(T^{\#}-z\right)=\operatorname{ker}\left(T^{\#}+z^{*}\right), \quad \forall z \in \sigma_{\mathrm{p}}\left(T^{\#}\right) .
$$

Proof. It is easy to see that operator equality $J T^{\#} J=-T^{\#}$ holds $\left(J D\left(T^{\#}\right)=D\left(T^{\#}\right)\right)$. Hence, for all $z \in \mathbb{C}$, we have $J\left(T^{\#}-z\right) J=-\left(T^{\#}+z^{*}\right) \cdots(*)$. This implies that, if $z \in \rho\left(T^{\#}\right)$, then $-z^{*} \in \rho\left(T^{\#}\right)$. Thus the same holds for the spectrum $\sigma\left(T^{\#}\right)=\mathbb{C} \backslash \rho\left(T^{\#}\right)$. Equation (3.8) follows from $(*)$. 


\section{Boundedness of $T$}

In this section we present a general criterion for the operator $T$ to be bounded. For mathematical generality and for later use, we consider a more general class of operators than that of $T$. Let $b:=\left\{b_{n m}\right\}_{n, m=1}^{\infty}$ be a double sequence of complex numbers such that

$$
\|b\|_{\infty}:=\sup _{n, m \geq 1}\left|b_{n m}\right|<\infty .
$$

Then, in the same way as in Lemma 2.1-(ii), for all $\psi \in \mathcal{H}$, the infinite series

$$
\sum_{m \neq n}^{\infty} \frac{b_{n m}}{E_{n}-E_{m}}\left\langle e_{m}, \psi\right\rangle
$$

absolutely converges. Hence one can define a linear operator $T_{b}$ on $\mathcal{H}$ as follows:

$$
\begin{aligned}
& D\left(T_{b}\right):=\left\{\left.\psi \in \mathcal{H}\left|\sum_{n=1}^{\infty}\right| \sum_{m \neq n}^{\infty} \frac{b_{n m}}{E_{n}-E_{m}}\left\langle e_{m}, \psi\right\rangle\right|^{2}<\infty\right\}, \\
& T_{b} \psi:=i \sum_{n=1}^{\infty}\left(\sum_{m \neq n}^{\infty} \frac{b_{n m}}{E_{n}-E_{m}}\left\langle e_{m}, \psi\right\rangle\right) e_{n}, \quad \psi \in D\left(T_{b}\right) .
\end{aligned}
$$

Obviously $T=T_{b}$ with $b$ satisfying $b_{n m}=1$ for all $n, m \in \mathbb{N}$. In the same way as in the case of $T$, one can prove the following fact:

Lemma 4.1 The operator $T_{b}$ is closed.

The following lemma is probably well known (but, for the completeness, we give a proof):

Lemma 4.2 Let $A$ be a densely defined linear operator on a Hilbert space $\mathcal{K}$. Suppose that there exist a dense subspace $\mathcal{D}$ in $\mathcal{K}$ and a constant $C>0$ such that $\mathcal{D} \subset D(A)$ and

$$
|\langle\psi, A \psi\rangle| \leq C\|\psi\|^{2}, \quad \psi \in \mathcal{D} .
$$

Then $A$ is bounded with $\|\bar{A}\| \leq 2 C$, where $\bar{A}$ is the closure of $A$.

If $A$ is symmetric in addition, then $\|\bar{A}\| \leq C$.

Proof. Let $\psi, \phi \in \mathcal{D}$. Then, by the polarization identity

$$
\begin{aligned}
\langle\psi, A \phi\rangle= & \frac{1}{4}(\langle\psi+\phi, A(\psi+\phi)\rangle-\langle\psi-\phi, A(\psi-\phi)\rangle \\
& +i\langle\psi-i \phi, A(\psi-i \phi)\rangle-i\langle\psi+i \phi, A(\psi+i \phi)\rangle),
\end{aligned}
$$

we have

$$
|\langle\psi, A \phi\rangle| \leq \frac{C}{4}\left(\|\psi+\phi\|^{2}+\|\psi-\phi\|^{2}+\|\psi-i \phi\|^{2}+\|\psi+i \phi\|^{2}\right)=C\left(\|\psi\|^{2}+\|\phi\|^{2}\right) .
$$


Replacing $\psi \neq 0$ by $\|\phi\| \psi /\|\psi\|$ we have

$$
|\langle\psi, A \phi\rangle| \leq 2 C\|\psi\|\|\phi\| \text {. }
$$

For $\psi=0$, this inequality trivially holds. Since $\mathcal{D}$ is dense, it follows from the Riesz representation theorem that $\|A \phi\| \leq 2 C\|\phi\|, \phi \in \mathcal{D}$. Thus the first half of the lemma follows.

Let $A$ be symmetric. Then, $\langle\psi, A \psi\rangle \in \mathbb{R}$ for all $\psi \in D(A)$. Hecne

$$
|\Re\langle\psi, A \phi\rangle|=\frac{1}{4}|\langle\psi+\phi, A(\psi+\phi)\rangle-\langle\psi-\phi, A(\psi-\phi)\rangle| \leq \frac{C}{2}\left(\|\psi\|^{2}+\|\phi\|^{2}\right), \quad \psi \in \mathcal{D} .
$$

We write $\langle\psi, A \phi\rangle=|\langle\psi, A \phi\rangle| e^{i \theta}$ with $\theta \in \mathbb{R}$. Then $|\langle\psi, A \phi\rangle|=\left\langle e^{i \theta} \psi, A \phi\right\rangle$. Hence

$$
\begin{aligned}
|\langle\psi, A \phi\rangle| & =\Re\left\langle e^{i \theta} \psi, A \phi\right\rangle \leq \frac{C}{2}\left(\left\|e^{i \theta} \psi\right\|^{2}+\|\phi\|^{2}\right) \\
& =\frac{C}{2}\left(\|\psi\|^{2}+\|\phi\|^{2}\right) .
\end{aligned}
$$

Thus, in the same manner as above, we can obtain $|\langle\psi, A \phi\rangle| \leq C\|\psi\|\|\phi\|, \psi, \phi \in \mathcal{D}$.

The next lemma is easily proven by elementary calculus. Therefore we omit proof of it.

Lemma 4.3 For all $s>1$ and $n \geq 2$,

$$
\sum_{m=1}^{n-1} \frac{1}{n^{s}-m^{s}} \leq \frac{\log n}{n^{s-1}}+\frac{1}{s(n-1)^{s-1}} .
$$

Lemma 4.4 Let $s>1$. Then

$$
\sup _{n \geq 2}\left(\sum_{m=1}^{n-1} \frac{1}{n^{s}-m^{s}}\right)<\infty
$$

and

$$
\sup _{m \geq 1}\left(\sum_{n=m+1}^{\infty} \frac{1}{n^{s}-m^{s}}\right)<\infty
$$

Proof. Property (4.5) follows from Lemma 4.3.

To prove (4.6), we write

$$
\sum_{n=m+1}^{\infty} \frac{1}{n^{s}-m^{s}} \leq \int_{m+1}^{\infty} \frac{1}{x^{s}-m^{s}} d x+\frac{1}{(m+1)^{s}-m^{s}}
$$

We fix a constant $R>2(\geq(m+1) / m)$. By the change of variable $x=m y$, we have

$$
\int_{m+1}^{\infty} \frac{1}{x^{s}-m^{s}} d x=\frac{1}{m^{s-1}}\left(\int_{(m+1) / m}^{R} \frac{1}{y^{s}-1} d s+C_{R}\right),
$$


where

$$
C_{R}:=\int_{R}^{\infty} \frac{1}{y^{s}-1} d s<\infty
$$

Using the well known inequality

$$
x^{s}-1 \geq s(x-1), \quad x>0, s \geq 1,
$$

we have

$$
\begin{aligned}
\int_{(m+1) / m}^{R} \frac{1}{y^{s}-1} d y & \leq \int_{(m+1) / m}^{R} \frac{1}{s(y-1)} d y \\
& =\frac{1}{s}(\log (R-1)+\log m)
\end{aligned}
$$

Hence

$$
\int_{m+1}^{\infty} \frac{1}{x^{s}-m^{s}} d x \leq \frac{\log m}{s m^{s-1}}+\frac{\log (R-1)}{s m^{s-1}}+\frac{1}{m^{s-1}} C_{R}
$$

Thus (4.6) follows.

Let

$$
\begin{aligned}
& c_{H}(n):=\sum_{m=1}^{n-1} \frac{E_{n}}{\left(E_{n}-E_{m}\right) E_{m}}, \quad n \geq 2, \\
& d_{H}(m):=\sum_{n=m+1}^{\infty} \frac{E_{m}}{\left(E_{n}-E_{m}\right) E_{n}}, \quad m \geq 1 .
\end{aligned}
$$

Since $c_{H}(n)$ and $d_{H}(m)$ are positive (recall that $E_{n}>0, \forall n \in \mathbb{N}$ ), one can define constants

$$
\begin{aligned}
c_{H} & :=\sup _{n \geq 2} c_{H}(n), \\
d_{H} & :=\sup _{m \geq 1} d_{H}(m),
\end{aligned}
$$

which are finite or infinite.

Theorem 4.5 Suppose that there exist constants $\alpha>1, C>0$ and $a>0$ such that

$$
E_{n}-E_{m} \geq C\left(n^{\alpha}-m^{\alpha}\right), \quad n>m>a .
$$

Then $T_{b}$ is a bounded operator with $D\left(T_{b}\right)=\mathcal{H}$ and

$$
\left\|T_{b}\right\| \leq 4\|b\|_{\infty} \sqrt{c_{H} d_{H}}
$$

Moreover, if $b_{n m}^{*}=b_{m n}$ for all $m, n \in \mathbb{N}$, then $T_{b}$ is a bounded self-adjoint operator with $D\left(T_{b}\right)=\mathcal{H}$ and

$$
\left\|T_{b}\right\| \leq 2\|b\|_{\infty} \sqrt{c_{H} d_{H}} .
$$

In particular, $T$ is a bounded self-adjoint operator with $D(T)=\mathcal{H}$. 
Proof. By Lemma 4.2, it is enough to show that $c_{H}$ and $d_{H}$ are finite and

$$
\left|\left\langle\psi, T_{b} \psi\right\rangle\right| \leq 2\|b\|_{\infty} \sqrt{c_{H} d_{H}}\|\psi\|^{2}, \quad \psi \in \mathcal{D}_{0} .
$$

Then $T_{b}$ is bounded with (4.13). Since $T_{b}$ is densely defined and closed, it follows that $D\left(T_{b}\right)=\mathcal{H}$. As in the case of $T$, one can show that, if $b_{n m}^{*}=b_{m n}$ for all $m, n \in \mathbb{N}$, then $T_{b} \mid \mathcal{D}_{0}$ is symmetric and hence $T_{b}$ is a bouned self-adjoint operator with $D\left(T_{b}\right)=\mathcal{H}$ and (4.14) holds. Therefore the desired result follows.

To prove (4.15), we first note that, for $\psi \in \mathcal{D}_{0}$,

$$
\left\langle\psi, T_{b} \psi\right\rangle=i \sum_{m, n=1, m \neq n}^{\infty} \frac{b_{n m}}{E_{n}-E_{m}}\left\langle\psi, e_{n}\right\rangle\left\langle e_{m}, \psi\right\rangle .
$$

Hence

$$
\left|\left\langle\psi, T_{b} \psi\right\rangle\right| \leq 2\|b\|_{\infty} A(\psi)
$$

where

$$
A(\psi):=\sum_{n>m \geq 1} \frac{\left|\left\langle e_{m}, \psi\right\rangle \|\left\langle\psi, e_{n}\right\rangle\right|}{E_{n}-E_{m}} .
$$

Inserting $1=\sqrt{E_{m} / E_{n}} \cdot \sqrt{E_{n} / E_{m}}$ into the summand on the right hand side and using the Cauchy-Schwarz inequality, we have

$$
A(\psi)^{2} \leq B(\psi) C(\psi)
$$

with

$$
\begin{aligned}
& B(\psi)=\sum_{n>m \geq 1} \frac{\left|\left\langle e_{n}, \psi\right\rangle\right|^{2}}{E_{n}-E_{m}} \cdot \frac{E_{n}}{E_{m}}, \\
& C(\psi)=\sum_{n>m \geq 1} \frac{\left|\left\langle e_{m}, \psi\right\rangle\right|^{2}}{E_{n}-E_{m}} \cdot \frac{E_{m}}{E_{n}} .
\end{aligned}
$$

One can rewrite and estimate $B(\psi)$ as follows:

$$
\begin{aligned}
B(\psi) & =\sum_{n=2}^{\infty}\left|\left\langle e_{n}, \psi\right\rangle\right|^{2} c_{H}(n) \\
& \leq\|\psi\|^{2} c_{H} .
\end{aligned}
$$

Similarly we have

$$
C(\psi) \leq\|\psi\|^{2} d_{H}
$$

Hence

$$
\left|\left\langle\psi, T_{b} \psi\right\rangle\right| \leq 2\|b\|_{\infty} \sqrt{c_{H} d_{H}}\|\psi\|^{2} .
$$

Therefore we need only to prove that $c_{H}$ and $d_{H}$ are finite. 
We can write

$$
c_{H}(n)=\sum_{m=1}^{n-1} \frac{1}{E_{n}-E_{m}}+\sum_{m=1}^{n-1} \frac{1}{E_{m}} .
$$

By assumption (4.12), we have

$$
\frac{1}{E_{n}-E_{m}} \leq \frac{1}{C\left(n^{\alpha}-m^{\alpha}\right)}, \quad n>m>a .
$$

Since we have

$$
\sum_{n=1}^{\infty} \frac{1}{n^{\alpha}}<\infty
$$

it follows that

$$
\varepsilon_{1}:=\sum_{m=1}^{\infty} \frac{1}{E_{m}}<\infty
$$

Thus

$$
c_{H}(n) \leq \sum_{m=1}^{n-1} \frac{1}{E_{n}-E_{m}}+\varepsilon_{1} .
$$

Let $n_{0} \geq 2$ be a natural number such that $n_{0}>a$. Then, for all $n>n_{0}$

$$
\sum_{m=1}^{n-1} \frac{1}{E_{n}-E_{m}} \leq \sum_{m=1}^{n_{0}-1} \frac{1}{E_{n}-E_{m}}+\frac{1}{C} \sum_{m=n_{0}}^{n-1} \frac{1}{n^{\alpha}-m^{\alpha}} .
$$

By (4.4), the right hand side is uniformly bounded in $n$. Thus we have $c_{H}<\infty$.

To prove $d_{H}<\infty$, we write for $m>a$

$$
\begin{aligned}
d_{H}(m) & =\sum_{n=m+1}^{\infty} \frac{1}{\left(E_{n}-E_{m}\right)}-\sum_{n=m+1}^{\infty} \frac{1}{E_{n}} \\
& \leq \sum_{n=m+1}^{\infty} \frac{1}{\left(E_{n}-E_{m}\right)} \\
& \leq \frac{1}{C} \sum_{n=m+1}^{\infty} \frac{1}{n^{\alpha}-m^{\alpha}} .
\end{aligned}
$$

Hence, by (4.6) in Lemma 4.4, we have

$$
\sup _{m>a} d_{H}(m)<\infty
$$

Thus it follows that $d_{H}<\infty$.

Example 4.1 Let $\lambda>0, \alpha>1$ and $P(x)$ be a real polynomial of $x \in \mathbb{R}$ with degree $p<\alpha$. Then it is easy to see that the sequense $\left\{E_{n}\right\}_{n=1}^{\infty}$ defined by

$$
E_{n}:=\lambda n^{\alpha}+P(n)
$$

satisfies the assumptions (H.1), (H.2) and (4.12). Thus, by Theorem 4.5, in the present example, $T_{b}$ is bounded. 
We remark that Theorem 4.5 does not cover the case $E_{n}=\lambda n+\mu$ with constants $\lambda>0$ and $\mu \in \mathbb{R}$. For this case, we have the following theorem:

Theorem 4.6 Suppose that there exist constants $\lambda>0, \mu \in \mathbb{R}$ and $a>0$ such that

$$
E_{n}=\lambda n+\mu, \quad n>a .
$$

Then $T$ is a bounded self-adjoint operator with $D(T)=\mathcal{H}$.

Proof. Let $k_{0}$ be the greatest integer such that $k_{0} \leq a$. Let $a_{n}:=\left\langle e_{n}, \psi\right\rangle(\psi \in \mathcal{H})$. Then, by the Parseval equality, we have $\sum_{n=1}^{\infty}\left|a_{n}\right|^{2}=\|\psi\|^{2}$. Let $\psi \in \mathcal{D}_{0}$. Then we can write:

$$
\langle\psi, T \psi\rangle=S_{I}+S_{2}+S_{3}+S_{4}
$$

where

$$
\begin{aligned}
S_{1} & :=i \sum_{n=1}^{k_{0}} \sum_{m \neq n}^{k_{0}} \frac{a_{n}^{*} a_{m}}{E_{n}-E_{m}}, \\
S_{2} & :=i \sum_{n=1}^{k_{0}} \sum_{m \geq k_{0}+1}^{\infty} \frac{a_{n}^{*} a_{m}}{E_{n}-E_{m}}, \\
S_{3} & :=i \sum_{n \geq k_{0}+1}^{\infty} \sum_{m=1}^{k_{0}} \frac{a_{n}^{*} a_{m}}{E_{n}-E_{m}}, \\
S_{4} & :=i \frac{1}{\lambda} \sum_{n=k_{0}+1}^{\infty} \sum_{m \neq n, m \geq k_{0}+1}^{\infty} \frac{a_{n}^{*} a_{m}}{n-m} .
\end{aligned}
$$

By the Schwarz inequality, we have

$$
\left|S_{j}\right| \leq C_{j}\|\psi\|^{2}, \quad j=1,2,3,
$$

where $C_{j}>0$ is a constant. To estimate $\left|S_{4}\right|$, we use the following well known inequality [11, Theorem 294]:

$$
\left|\sum_{n, m=1, n \neq m}^{\infty} \frac{x_{n} y_{m}}{n-m}\right| \leq \pi \sqrt{\sum_{n=1}^{\infty} x_{n}^{2}} \sqrt{\sum_{m=1}^{\infty} y_{m}^{2}}
$$

for all real sequences $\left\{x_{n}\right\}_{n=1}^{\infty}$ and $\left\{y_{n}\right\}_{n=1}^{\infty}$. Hence

$$
\left|S_{4}\right| \leq \pi\|\psi\|^{2} .
$$

Therefore it follows that $|\langle\psi, T \psi\rangle| \leq$ const. $\|\psi\|^{2}$. Thus $T$ is bounded.

Example 4.2 A physically interesting case is the case where $E_{n}=\omega\left(n+\frac{1}{2}\right), \quad n \in\{0\} \cup \mathbb{N}$ with a constant $\omega>0$. In this case, by Theorem 4.6, $T$ is a bounded self-adjoint operator with $D(T)=\mathcal{H}$ and takes the form

$$
T \psi=\frac{i}{\omega} \sum_{n=1}^{\infty}\left(\sum_{m \neq n}^{\infty} \frac{\left\langle e_{m}, \psi\right\rangle}{n-m}\right) e_{n}, \quad \psi \in \mathcal{H} .
$$


Moreover, one can prove that

$$
\sigma(T)=[-\pi / \omega, \pi / \omega]
$$

([4, Theorem 2.1]).

Let $\hat{N}:=\omega^{-1} H-1 / 2$ and $\hat{\theta}:=\omega T$. Then it follows that

$$
\begin{aligned}
& \sigma(\hat{N})=\{0\} \cup \mathbb{N}, \quad \sigma(\hat{\theta})=[-\pi, \pi], \\
& {[\hat{\theta}, \hat{N}] \psi=i \psi, \quad \psi \in \mathcal{D}_{\mathrm{c}} .}
\end{aligned}
$$

As is well known, in the context of quantum mechanics, the sequence $\left\{\omega\left(n+\frac{1}{2}\right)\right\}_{n=1}^{\infty}$ appears as the spectrum of the one-dimensional quantum harmonic oscillator Hamiltonian with mass $m>0$

$$
H_{\mathrm{os}}:=\frac{p^{2}}{2 m}+\frac{1}{2} m \omega^{2} q^{2}
$$

in the Schrödinger representation $(q, p)$ of the CCR, where $p:=-i D$ with $D$ being the generalized partial differential operator on $L^{2}(\mathbb{R})$ and $q$ is the multiplication operator by the variable $x \in \mathbb{R}$. In this context, the operator $\hat{N}$ is called the number operator and, in view of (4.20) and (4.21), the operator $\hat{\theta}$ is interpreted as a phase operator [7].

\section{$5 \quad$ Unboundedness of $T$}

As for the unboundedness of $T$, we have the following fact:

Theorem 5.1 If $\left\{E_{n}\right\}_{n=1}^{\infty}$ satisfies

$$
\inf _{n \in \mathbb{N}}\left(E_{n+1}-E_{n}\right)=0
$$

then $T$ is unbounded.

Proof. By (5.1), there exists a subsequence $\left\{E_{p_{k}}\right\}_{k=1}^{\infty}$ of $\left\{E_{p}\right\}_{p=1}^{\infty}$ such that

$$
\lim _{k \rightarrow \infty}\left(E_{p_{k}+1}-E_{p_{k}}\right)=0 .
$$

Hence we have

$$
\begin{aligned}
\left\|T e_{p_{k}}\right\|^{2} & =\sum_{n=1}^{\infty}\left|\sum_{m \neq n}^{\infty} \frac{\left\langle e_{m}, e_{p_{k}}\right\rangle}{E_{n}-E_{m}}\right|^{2}=\sum_{n \neq p_{k}}^{\infty}\left|\frac{1}{E_{n}-E_{p_{k}}}\right|^{2} \\
& \geq\left|\frac{1}{E_{p_{k}+1}-E_{p_{k}}}\right|^{2} \rightarrow \infty(k \rightarrow \infty) .
\end{aligned}
$$

Thus $T$ is unbounded.

Example 5.1 Let

$$
E_{n}=\lambda n^{\alpha}+\mu
$$

with constants $\lambda>0, \alpha>1 / 2$ and $\mu \in \mathbb{R}$. Then $\left\{E_{n}\right\}_{n=1}^{\infty}$ satisfies the assumptions (H.1) and (H.2). As we have already seen, $T$ is bounded if $\alpha \geq 1$ 
Let $1 / 2<\alpha<1$. Then one easily sees that

$$
\lim _{n \rightarrow \infty}\left(E_{n+1}-E_{n}\right)=0 .
$$

Hence $\inf _{n \in \mathbb{N}}\left(E_{n+1}-E_{n}\right)=0$. Therefore, in this case, $T$ is unbounded. Thus $T$ is bounded if and only if $\alpha \geq 1$.

\section{$6 \quad$ Hilbert-Schmidtness of $T$}

In this section we investigate Hilbert-Schmidtness of the operator $T$.

Proposition 6.1 The operator $T$ is Hilbert-Schmidt if and only if

$$
\sum_{n=1}^{\infty} \sum_{m \neq n}^{\infty} \frac{1}{\left(E_{n}-E_{m}\right)^{2}}<\infty
$$

In that case, $T$ is self-adjoint with

$$
\|T\|_{2}^{2}=\sum_{n=1}^{\infty} \sum_{m \neq n}^{\infty} \frac{1}{\left(E_{n}-E_{m}\right)^{2}},
$$

where $\|\cdot\|_{2}$ denotes Hilbert-Schmidt norm. In particular, there exist a C.O.N.S. $\left\{f_{n}\right\}_{n=1}^{\infty}$ of $\mathcal{H}$ and real numbers $t_{n}, n \in \mathbb{N}$ such that $T f_{n}=t_{n} f_{n}$ and $t_{n} \rightarrow 0(n \rightarrow \infty)$.

Proof. Suppose that $T$ is Hilbert-Schmidt. Then $\sum_{n=1}^{\infty}\left\|T e_{n}\right\|^{2}<\infty$. On the other hand, we have

$$
\sum_{n=1}^{\infty}\left\|T e_{n}\right\|^{2}=\sum_{n=1}^{\infty} \sum_{m \neq n}^{\infty} \frac{1}{\left(E_{n}-E_{m}\right)^{2}}
$$

Hence (6.1) follows with (6.2).

Conversely, (6.1) holds. Hence, by (6.3), $\sum_{n=1}^{\infty}\left\|T e_{n}\right\|^{2}<\infty$. Therefore $T$ is HilbertSchmidt. The last statement follows from the Hilbert-Schmidt theorem (e.g., [14, Theorem VI.16]).

Remark 6.1 In Proposition 6.1, the number $t_{n} \neq 0$ is an eigenvalue of $T$ with a finite multiplicity. Since $T$ is self-adjoint in the present case, it may be an observable in the context of quantum mechanics. If this is the case, then Proposition 6.1 shows that the observable described by $T$ ("time" in any sense ?) is quantized (discretized) in the quantum system whose Hamiltonian is $H$ with eigenvalues $\left\{E_{n}\right\}_{n=1}^{\infty}$ satisfying (6.1).

The next theorem gives a class of $H$ such that $T$ is Hilbert-Schmidt:

Theorem 6.2 Suppose that (4.12) in Theorem 4.5 holds with $\alpha>3 / 2$. Then $T$ is HilbertSchmidt and self-adjoint. 
Proof. Since $1 /\left(E_{n}-E_{m}\right)^{2}$ is symmetric in $n$ and $m$, it is sufficient to show that $\sum_{n>m \geq 1} 1 /\left(E_{n}-E_{m}\right)^{2}<\infty$. By the present assumption, we need only to show that

$$
\Sigma:=\sum_{n>m \geq 1} \frac{1}{\left(n^{\alpha}-m^{\alpha}\right)^{2}}<\infty
$$

for all $\alpha>3 / 2$. We have

$$
\begin{aligned}
\Sigma & =\sum_{n=2}^{\infty} \sum_{m=1}^{n-1} \frac{1}{\left(n^{\alpha}-m^{\alpha}\right)^{2}} \\
& \leq \sum_{n=2}^{\infty} \frac{1}{n^{\alpha}-(n-1)^{\alpha}} \cdot \sum_{m=1}^{n-1} \frac{1}{\left(n^{\alpha}-m^{\alpha}\right)}
\end{aligned}
$$

Using (4.4) and the elementary inequality

$$
\frac{1}{n^{\alpha}-(n-1)^{\alpha} s} \leq \frac{1}{\alpha(n-1)^{\alpha-1}}
$$

we obtain

$$
\Sigma \leq \sum_{n=2}^{\infty} \frac{1}{\alpha(n-1)^{\alpha-1}} \frac{\log n}{n^{\alpha-1}}+\sum_{n=2}^{\infty} \frac{1}{\alpha^{2}(n-1)^{2(\alpha-1)}} .
$$

Each infinite series on the right hand side converges for all $\alpha>3 / 2$. Thus the desired result follows.

\section{The Galapon Time Operator as a Generalized Time Operator}

It is shown that every self-adjoint operator which has a strong time operator is absolutely continuous $[12,17]$. Hence the Galapon time operator $T_{1}$ is not a strong time operator of $H$. But it may be a generalized time operator of $H$. In this section we investigate this aspect.

\subsection{An operator-valued function on $\mathbb{R}$}

In the same way as in Lemma 2.1-(ii), one can show that, for all $\psi \in \mathcal{H}, n \in \mathbb{N}$ and all $t \in \mathbb{R}$, the infinite series

$$
\sum_{m \neq n}^{\infty} \frac{e^{i t\left(E_{n}-E_{m}\right)}-1}{E_{n}-E_{m}}\left\langle e_{m}, \psi\right\rangle
$$


absolutely converges. Hence, for each $t \in \mathbb{R}$, one can define a linear operator $K(t)$ as follows:

$$
\begin{aligned}
& D(K(t)):=\left\{\left.\psi \in \mathcal{H}\left|\sum_{n=1}^{\infty}\right| \sum_{m \neq n}^{\infty} \frac{e^{i t\left(E_{n}-E_{m}\right)}-1}{E_{n}-E_{m}}\left\langle e_{m}, \psi\right\rangle\right|^{2}<\infty\right\}, \\
& K(t) \psi:=i \sum_{n=1}^{\infty}\left(\sum_{m \neq n}^{\infty} \frac{e^{i t\left(E_{n}-E_{m}\right)}-1}{E_{n}-E_{m}}\left\langle e_{m}, \psi\right\rangle\right) e_{n}, \quad \psi \in D(K(t)) .
\end{aligned}
$$

It is easy to see that, for all $t \in \mathbb{R}$,

$$
\mathcal{D}_{0} \subset D(K(t))
$$

and

$$
K(t) e_{k}=i \sum_{n \neq k} \frac{e^{i t\left(E_{n}-E_{k}\right)}-1}{E_{n}-E_{k}} e_{n}, \quad k \in \mathbb{N} .
$$

The correspondence $K: \mathbb{R} \ni t \mapsto K(t)$ gives an operator-valued function on $\mathbb{R}$. In the notation in Section $4, K(t)$ is the operator $T_{b}$ with $b_{n m}=e^{i t\left(E_{n}-E_{m}\right)}-1, n, m \in \mathbb{N}$.

Remark 7.1 Equation (7.4) shows that $K(t) \neq t I \mid \mathcal{D}_{0}$. Hence $T$ cannot be a strong time operator of $H$, as already remarked based on the general theory of strong time operators.

Proposition 7.1 For all $t \in \mathbb{R}, K(t)$ is a densely defined closed operator.

Proof. Similar to the proof of Proposition 3.2.

Proposition 7.2 For all $t \in \mathbb{R}, K(t) \mid \mathcal{D}_{0}$ is symmetric.

Proof. Similar to the proof of Lemma 2.3.

Theorem 7.3 For all $\psi \in D\left(T_{1}\right)\left(=\mathcal{D}_{0}\right)$ and $t \in \mathbb{R}, e^{-i t H} \psi \in D\left(T_{1}\right)$ and

$$
T_{1} e^{-i t H} \psi=e^{-i t H}\left(T_{1}+K(t)\right) \psi \text {. }
$$

Proof. We need only to prove the statement in the case $\psi=e_{k}(\forall k \in \mathbb{N})$. Since $e^{-i t H} e_{k}=e^{-i t E_{k}} e_{k}$, it follows that $e^{-i t H} e_{k} \in D\left(T_{1}\right)$ with

$$
T_{1} e^{-i t H} e_{k}=e^{-i t E_{k}} \sum_{n \neq k}^{\infty} \frac{i}{E_{n}-E_{k}} e_{n} .
$$

We have

$$
e^{-i t H} T_{1} e_{k}=i \sum_{n \neq k}^{\infty} \frac{e^{-i t E_{n}}}{E_{n}-E_{k}} e_{n} .
$$

It follows from these equations that

$$
T_{1} e^{-i t H} e_{k}-e^{-i t H} T_{1} e_{k}=e^{-i t H} K(t) e_{k} .
$$

Thus the desired result follows. 
Corollary 7.4 Suppose that, for all $t \in \mathbb{R}, K(t)$ is bounded. Then $T_{1}$ is a generalized time operator of $H$ with commutation factor $K$.

Proof. This follows from Theorem 7.3, Proposition 7.1 and Proposition 7.2.

In view of Corollary 7.4, we need to investigate conditions for $K(t)$ to be bounded.

Proposition 7.5 Suppose that (4.12) holds with $\alpha>1$. Then, for all $t \in \mathbb{R}, K(t)$ is a bounded self-adjoint operator with $D(K(t))=\mathcal{H}$.

Proof. This follows from an application of Theorem 4.5 to the case where $b_{n m}=$ $e^{i t\left(E_{n}-E_{m}\right)}-1, n, m \in \mathbb{N}$.

Proposition 7.6 Suppose that (6.1) holds. Then, for all $t \in \mathbb{R}, K(t)$ is Hilbert-Schmidt and self-adjoint with

$$
\|K(t)\|_{2}^{2}=\sum_{k=1}^{\infty} \sum_{n \neq k}^{\infty}\left|\frac{e^{i t\left(E_{n}-E_{k}\right)}-1}{E_{n}-E_{k}}\right|^{2} .
$$

Proof. Similar to the proof of Proposition 6.1.

\section{$7.2 \quad$ Non-differentiability of $K$}

From the view-point of the theory of generalized time operators [2], it is interesting to examine differentiability of the operator-valued function $K$.

Proposition 7.7 For all $k \in \mathbb{N}$, the $\mathcal{H}$-valued function $: \mathbb{R} \ni t \mapsto K(t) e_{k}$ is not strongly differentiable on $\mathbb{R}$.

Proof. We first show that $K(t) e_{k}$ is not strongly differentiable at $t=0$. Since $K(0) e_{k}=$ 0 , we have for all $t \in \mathbb{R} \backslash\{0\}$ and $N>k$

$$
\begin{aligned}
\left\|\frac{K(t) e_{k}-K(0) e_{k}}{t}\right\|^{2} & =\sum_{n \neq k}^{\infty} \frac{\left|e^{i t\left(E_{n}-E_{k}\right)}-1\right|^{2}}{t^{2}\left|E_{n}-E_{k}\right|^{2}} \\
& \geq \sum_{n \neq k}^{N+1} \frac{\left|e^{i t\left(E_{n}-E_{k}\right)}-1\right|^{2}}{t^{2}\left|E_{n}-E_{k}\right|^{2}} .
\end{aligned}
$$

Hence

$$
\liminf _{t \rightarrow 0}\left\|\frac{K(t) e_{k}-K(0) e_{k}}{t}\right\|^{2} \geq \sum_{n \neq k}^{N+1} 1=N .
$$

Since $N>k$ is arbitrary, it follows that

$$
\lim _{t \rightarrow 0}\left\|\frac{K(t) e_{k}-K(0) e_{k}}{t}\right\|^{2}=+\infty .
$$


This implies that $K(t) e_{k}$ is not strongly differentiable at $t=0$.

We next show that $K(t) e_{k}$ is not strongly differentiable at each $t \neq 0$. By (7.5), we have for all $s \in \mathbb{R} \backslash\{0\}$

$$
\frac{K(t+s) e_{k}-K(t) e_{k}}{s}=e^{i t\left(H-E_{k}\right)} \frac{K(s) e_{k}}{s} .
$$

Hence

$$
\left\|\frac{K(t+s) e_{k}-K(t) e_{k}}{s}\right\|=\left\|\frac{K(s) e_{k}}{s}\right\| .
$$

By the preceding result, the right hand side diverges to $+\infty$ as $s \rightarrow 0$. Therefore $K(t) e_{k}$ is not strongly differentiable at $t$.

Remark 7.2 We have

$$
\left\langle e_{\ell}, K(t) e_{k}\right\rangle= \begin{cases}i \frac{e^{i t\left(E_{\ell}-E_{k}\right)}-1}{E_{\ell}-E_{k}} & ; \ell \neq k \\ 0 & ; \ell=k\end{cases}
$$

Hence, for all $k, \ell \in \mathbb{N},\left\langle e_{\ell}, K(t) e_{k}\right\rangle$ is differentiable in $t \in \mathbb{R}$ and

$$
\frac{d}{d t}\left\langle e_{\ell}, K(t) e_{k}\right\rangle=\left(\delta_{\ell k}-1\right) e^{i t\left(E_{\ell}-E_{k}\right)}
$$

Proposition 7.7 tells us some singularity of $K(t)$ acting on $\mathcal{D}_{0}$. But, as shown in the next proposition, $K(t)$ restricted to $\mathcal{D}_{c}$ is strongly differentiable at $t=0$.

Proposition 7.8 For all $\psi \in \mathcal{D}_{c}$, the $\mathcal{H}$-valued function $K(t) \psi$ is strongly differentiable at $t=0$ with

$$
\left.\frac{d}{d t} K(t) \psi\right|_{t=0}=\psi
$$

Proof. We need only to prove the statement for $\psi$ of the form $\psi=e_{k}-e_{\ell}(k, \ell \in$ $\mathbb{N}, k \neq \ell$ ). For all $t \in \mathbb{R} \backslash\{0\}$, we have

$$
\frac{K(t)\left(e_{k}-e_{\ell}\right)}{t}=A(t)+B(t)
$$

where

$$
\begin{aligned}
& A(t):=i \frac{e^{i t\left(E_{\ell}-E_{k}\right)}-1}{t\left(E_{\ell}-E_{k}\right)} e_{\ell}-i \frac{e^{i t\left(E_{k}-E_{\ell}\right)}-1}{t\left(E_{k}-E_{\ell}\right)} e_{k}, \\
& B(t):=i \sum_{n \neq k, \ell}^{\infty}\left(\frac{e^{i t\left(E_{n}-E_{k}\right)}-1}{t\left(E_{n}-E_{k}\right)}-\frac{e^{i t\left(E_{n}-E_{\ell}\right)}-1}{t\left(E_{n}-E_{\ell}\right)}\right) e_{n} .
\end{aligned}
$$

It is easy to see that

$$
\lim _{t \rightarrow 0} A(t)=e_{k}-e_{\ell}
$$


As for $B(t)$, we have

$$
\|B(t)\|^{2}=\sum_{n \neq k, \ell}^{\infty}\left|F_{n}(t)\right|^{2},
$$

where

$$
F_{n}(t):=\frac{e^{i t\left(E_{n}-E_{k}\right)}-1}{t\left(E_{n}-E_{k}\right)}-\frac{e^{i t\left(E_{n}-E_{\ell}\right)}-1}{t\left(E_{n}-E_{\ell}\right)}
$$

It is easy to see that

$$
\lim _{t \rightarrow 0} F_{n}(t)=0
$$

Moreover, one can show that

$$
\left|F_{n}(t)\right| \leq \frac{C}{\left|E_{n}-E_{k}\right|}, \quad n \neq k, \ell
$$

where $C>0$ is a constant independent of $n$ and $t$. Since $\sum_{n \neq k}^{\infty} 1 /\left|E_{n}-E_{k}\right|^{2}<\infty$, one can apply the dominated convergence theorem to conclude that $\lim _{t \rightarrow 0}\|B(t)\|^{2}=0$. Thus $K(t)\left(e_{k}-e_{\ell}\right)$ is strongly differentiable at $t=0$ and (7.9) with $\psi=e_{k}-e_{\ell}$ holds.

Proposition 7.9 For all $k, \ell \in \mathbb{N}$ with $k \neq \ell$, the $\mathcal{H}$-valued function $K(t)\left(e_{k}-e_{\ell}\right)$ is not strongly differentiable at $t \notin\left\{2 \pi n /\left(E_{k}-E_{\ell}\right) \mid n \in \mathbb{Z}\right\}$.

Proof. Let $t \neq 2 \pi n /\left(E_{k}-E_{\ell}\right)(n \in \mathbb{Z})$ and $s \in \mathbb{R} \backslash\{0\}$. Then, by (7.5), we have

$$
\frac{(K(t+s)-K(t))\left(e_{k}-e_{\ell}\right)}{s}=e^{i t H} \frac{K(s)}{s} e^{-i t H}\left(e_{k}-e_{\ell}\right) .
$$

Hence

$$
\left\|\frac{(K(t+s)-K(t))\left(e_{k}-e_{\ell}\right)}{s}\right\|=\|u(s)\|
$$

with

$$
u(s):=\frac{K(s)}{s}\left(e^{-i t E_{k}} e_{k}-e^{-i t E_{\ell}} e_{\ell}\right)
$$

We write

$$
u(s)=u_{1}(s)+u_{2}(s)
$$

with

$$
u_{1}(s):=e^{-i t E_{k}} \frac{K(s)}{s}\left(e_{k}-e_{\ell}\right), \quad u_{2}(s):=\left(e^{-i t E_{k}}-e^{-i t E_{\ell}}\right) \frac{K(s)}{s} e_{\ell} .
$$

By Proposition 7.8, we have $\lim _{s \rightarrow 0} u_{1}(s)=e^{-i t E_{k}}\left(e_{k}-e_{\ell}\right)$. On the other hand, we have from the proof of Proposition 7.7 and the assumed condition for $t$

$$
\lim _{s \rightarrow 0}\left\|u_{2}(s)\right\|=+\infty
$$

Hence $\lim _{s \rightarrow 0}\|u(s)\|=+\infty$. Thus the desired result follows. 


\section{Possible Connections with Regular Perturbation Theory}

We consider a perturbation of $H$ by a symmetric operator $H_{I}$ on $\mathcal{H}$ :

$$
H(\lambda):=H+\lambda H_{I}
$$

where $\lambda \in \mathbb{R}$ is a perturbation parameter. For simplicity, we assume that $H_{I}$ is $H$ bounded: $D(H) \subset D\left(H_{I}\right)$ and there exist constants $a, b \geq 0$ such that

$$
\left\|H_{I} \psi\right\| \leq a\|H \psi\|+b\|\psi\|, \quad \psi \in D(H) .
$$

Then, by the Kato-Rellich theorem (e.g., [15, Theorem X.12]), for all $\lambda \in \mathbb{R}$ satisfying

$$
a|\lambda|<1
$$

$H(\lambda)$ is self-adjoint and bounded below. In what follows, we assume (8.2).

\subsection{Eigenvalues of $H(\lambda)$}

We fix $n \in \mathbb{N}$ arbitrarily. By a general theorem in regular perturbation theory (e.g., [16, Theorem XII.9]), there exists a constant $c_{n}>0$ such that, for all $|\lambda|<c_{n}, H$ has a unique, isolated non-degenerate eigenvalue $E_{n}(\lambda)$ near $E_{n}$. Moreover, $E_{n}(\lambda)$ is analytic in $\lambda$ with Taylor expansion

$$
E_{n}(\lambda)=E_{n}+E_{n}^{(1)} \lambda+E_{n}^{(2)} \lambda^{2}+\cdots
$$

where

$$
E_{n}^{(1)}:=\left\langle e_{n}, H_{I} e_{n}\right\rangle, \quad E_{n}^{(2)}:=\sum_{m \neq n}^{\infty} \frac{\left|\left\langle e_{n}, H_{I} e_{m}\right\rangle\right|^{2}}{E_{n}-E_{m}} .
$$

As an eigenvector of $H(\lambda)$ with eigenvalue $E_{n}(\lambda)$, one can take a vector $\psi_{n}(\lambda)$ analytic in $\lambda$ with Taylor expansion

$$
\psi_{n}(\lambda)=e_{n}+e_{n}^{(1)} \lambda+\cdots,
$$

where

$$
e_{n}^{(1)}:=\sum_{m \neq n}^{\infty} \frac{\left\langle e_{m}, H_{I} e_{n}\right\rangle}{E_{n}-E_{m}} e_{m} .
$$

By Lemma 2.2, we have

$$
\left\langle e_{n}, T e_{m}\right\rangle= \begin{cases}\frac{i}{E_{n}-E_{m}} & ; n \neq m \\ 0 & ; n=m\end{cases}
$$

Hence $E_{n}^{(2)}$ can be written

$$
E_{n}^{(2)}=(-i) \sum_{m=1}^{\infty}\left|\left\langle e_{n}, H_{I} e_{m}\right\rangle\right|^{2}\left\langle e_{n}, T e_{m}\right\rangle
$$


To rewrite the right hand side only in terms of $e_{n}$ and linear operators on $\mathcal{H}$, we note that

$$
\sum_{m=1}^{\infty}\left|\left\langle e_{n}, H_{I} e_{m}\right\rangle\right|^{2}=\left\|H_{I} e_{n}\right\|^{2}<\infty
$$

by the Parseval equality. Hence

$$
\sum_{m=1}^{\infty}\left|\left\langle e_{n}, H_{I} e_{m}\right\rangle\right|^{4}<\infty
$$

Therefore the infinite series

$$
f_{n}:=\sum_{m=1}^{\infty}\left|\left\langle e_{n}, H_{I} e_{m}\right\rangle\right|^{2} e_{m}
$$

strongly converges and defines a vector in $\mathcal{H}$. Thus we can define a linear operator $V$ on $\mathcal{H}$ as follows:

$$
\begin{aligned}
& D(V):=\mathcal{D}_{0} \\
& V \psi:=-i \sum_{n=1}^{\infty}\left\langle e_{n}, \psi\right\rangle f_{n}, \quad \psi \in \mathcal{D}_{0}
\end{aligned}
$$

where the right hand side of (8.10) is a sum over a finite term. It is easy to see that $V$ is a symmetric operator.

Proposition 8.1 For all $n \in \mathbb{N}$,

$$
E_{n}^{(2)}=\left\langle T e_{n}, V e_{n}\right\rangle
$$

Proof. We have $V e_{n}=-i f_{n}$. Hence $\left\langle T e_{n}, V e_{n}\right\rangle=-i\left\langle T e_{n}, f_{n}\right\rangle$, which is equal to the right hand side of (8.7).

This proposition suggests some role of the time operator $T_{1}=T \mid \mathcal{D}_{0}$ in the perturbation expansions of the eigenvalues of $H$.

As for the first order term $e_{n}^{(1)} \lambda$ of the eigenvector $\psi_{n}(\lambda)$, we have

$$
e_{n}^{(1)}=(-i) \sum_{m=1}^{\infty}\left\langle e_{m}, H_{I} e_{n}\right\rangle\left\langle e_{n}, T e_{m}\right\rangle e_{m}
$$

\subsection{Transition probability amplitudes}

In the context of quantum mechanics where $H(\lambda)$ is the Hamiltonian of a quantum system, the complex number $\left\langle\phi, e^{-i t H(\lambda)} \psi\right\rangle$ with unit vectors $\phi, \psi \in \mathcal{H}$ is called the transition probability amplitude for the probability such that the state of the quantum system at time $t$ is found in the state $\phi$ under the condition that the state of the quantum system at time zero is $\psi$. 
Lemma 8.2 Let $\phi, \psi \in D(H)$. Then, for all $t \in \mathbb{R}$,

$$
\left\langle\phi, e^{-i t H(\lambda)} \psi\right\rangle=\left\langle\phi, e^{-i t H} \psi\right\rangle-i \lambda \int_{0}^{t}\left\langle e^{i(t-s) H} \phi, H_{I} e^{-i s H} \psi\right\rangle d s+O\left(\lambda^{2}\right) .
$$

Proof. By a simple application of a general formula for the unitary group generated by a self-adjoint operator ([5, Lemma 5.9]), we have

$$
e^{-i t H(\lambda)} \psi=e^{-i t H} \psi-i \lambda \int_{0}^{t} e^{-i(t-s) H(\lambda)} H_{I} e^{-i s H} \psi d s,
$$

where the integral is taken in the strong sense. Hence

$$
\begin{aligned}
\left\langle\phi, e^{-i t H(\lambda)} \psi\right\rangle & =\left\langle\phi, e^{-i t H} \psi\right\rangle-i \lambda \int_{0}^{t}\left\langle e^{i(t-s) H(\lambda)} \phi, H_{I} e^{-i s H} \psi\right\rangle d s \\
& =\left\langle\phi, e^{-i t H} \psi\right\rangle-i \lambda \int_{0}^{t}\left\langle e^{i(t-s) H} \phi, H_{I} e^{-i s H} \psi\right\rangle d s+R(\lambda),
\end{aligned}
$$

where

$$
R(\lambda):=-i \lambda \int_{0}^{t}\left\langle\left(e^{i(t-s) H(\lambda)}-e^{i(t-s) H}\right) \phi, H_{I} e^{-i s H} \psi\right\rangle d s .
$$

Using (8.14) again, we have

$$
R(\lambda)=-\lambda^{2} \int_{0}^{t} d s \int_{0}^{-(t-s)} d s^{\prime}\left\langle e^{i\left(t-s+s^{\prime}\right) H(\lambda)} H_{I} e^{-i s^{\prime} H} \phi, H_{I} e^{-i s H} \psi\right\rangle .
$$

Hence

$$
|R(\lambda)| \leq \lambda^{2} \int_{0}^{|t|} d s \int_{0}^{|t-s|} d s^{\prime}\left\|H_{I} e^{-i s^{\prime} H} \phi\right\|\left\|H_{I} e^{-i s H} \psi\right\|
$$

Therefore $R(\lambda)=O\left(\lambda^{2}\right)$. Thus (8.13) holds.

Applying (8.13) with $\phi=e_{m}$ and $\psi=e_{n}(n \neq m)$, we have

$$
\left\langle e_{m}, e^{-i t H(\lambda)} e_{n}\right\rangle=-\lambda \frac{e^{-i t E_{n}}-e^{-i t E_{m}}}{E_{m}-E_{n}}\left\langle e_{m}, H_{I} e_{n}\right\rangle+O\left(\lambda^{2}\right),
$$

which, combined with (7.7), gives

$$
\left\langle e_{m}, e^{-i t H(\lambda)} e_{n}\right\rangle=i \lambda\left\langle e_{n}, e^{-i t H} K(t) e_{m}\right\rangle\left\langle e_{m}, H_{I} e_{n}\right\rangle+O\left(\lambda^{2}\right) . \quad m \neq n .
$$

This suggests a physical meaning of the commutation factor $K$.

By Theorem 7.3, one can rewrite the first term on the right hand side in terms of $T_{1}$ and $e^{-i t H}$, obtaining

$$
\left\langle e_{m}, e^{-i t H(\lambda)} e_{n}\right\rangle=i \lambda\left\langle e_{n},\left[T_{1}, e^{-i t H}\right] e_{m}\right\rangle\left\langle e_{m}, H_{I} e_{n}\right\rangle+O\left(\lambda^{2}\right), \quad m \neq n .
$$

This also is suggestive on physical meaning of the time operator $T_{1}$. 


\section{References}

[1] Y. Aharonov and D. Bohm, Time in the quantum theory and the uncertainty relation for time and energy, Phys. Rev. 122 (1961), 1649-1658.

[2] A. Arai, Generalized weak Weyl relation and decay of quantum dynamics, Rev. Math. Phys. 17 (2005) , 1071-1109 .

[3] A. Arai, Spectrum of time operators, Lett. Math. Phys. 80 (2007), 211-221.

[4] A. Arai, Some aspects of time operators, in "Quantum Bio-Informatics" (L. Accardi, W. Freudenberg and M. Ohya eds.), pp.26-35, 2008, World Scientific, Sigapore.

[5] A. Arai, Heisenberg operators, invariant domains and Heisenberg equations of motion, Rev. Math. Phys. 19 (2007), 1045-1069.

[6] A. Arai and Y. Matsuzawa, Construction of a Weyl representation from a weak Weyl representation of the canonical commutation relation, Lett. Math. Phys. 83 (2008), 201-211.

[7] G. Dorfmeister and J. Dorfmeister, Classification of certain pairs of operators $(P, Q)$ satisfying $[P, Q]=-i \mathrm{Id}$, J. Funct. Anal. 57 (1984), 301-328.

[8] E. A. Galapon, Pauli's theorem and quantum canonical pairs : the consistency of a bounded, self-adjoint time operator canonically conjugate to a Hamiltonian with non-empty point spectrum, Proc. R. Soc. Lond. A 458 (2002), 451-472.

[9] E. A. Galapon, Self-adjoint time operator is the rule for discrete semi-bounded Hamiltonians, Proc. R. Soc. Lond. A 458 (2002), 2671-2689.

[10] E. A. Galapon, R. F. Caballar and R. T. Bahague Jr, Confined quantum time of arrivals, Phys. Rev. Lett. 93 (2004), 180406.

[11] G. H. Hardy, J. E. Littlewood and G. Pólya, Inequalities (Cambridge University Press, London, 1934).

[12] M. Miyamoto, A generalized Weyl relation approach to the time operator and its connection to the survival probability, J. Math. Phys. 42 (2001), 1038-1052.

[13] J. von Neumann, Die Eindeutigkeit der Schrödingerschen Operatoren, Math. Ann. (1931) 104, 570-578.

[14] M. Reed and B. Simon, Methods of Modern Mathematical Physics I: Functional Analysis (Academic Press, New York, 1972).

[15] M. Reed and B. Simon, Methods of Modern Mathematical Physics II: Fourier Analysis, Self-adjointness (Academic Press, New York, 1975).

[16] M. Reed and B. Simon, Methods of Modern Mathematical Physics IV: Analysis of Operators (Academic Press, New York, 1978). 
[17] K. Schmüdgen, On the Heisenberg commutation relation. I, J. Funct. Anal. 50 (1983), 8-49.

[18] K. Schmüdgen, On the Heisenberg commutation relation. II, Publ. RIMS, Kyoto Univ. 19 (1983), 601-671. 